\title{
Bihemispheric Cortical Infarcts in an Adult Secondary to Escherichia coli K1 Meningitis
}

\author{
Bhanu Gogia ${ }^{a}$ Angela L. Young ${ }^{b}$ Barbara J. O'Brien ${ }^{c}$ \\ Pablo C. Okhuysen ${ }^{d}$ Vinodh A. Kumar ${ }^{b}$ \\ aDepartment of Neurology, University of Texas Medical Branch, Galveston, TX, USA; \\ ${ }^{b}$ Department of Neuroradiology, University of Texas MD Anderson Cancer Center, Houston, \\ TX, USA; ' ${ }^{2}$ epartment of Neuro-Oncology, University of Texas MD Anderson Cancer Center, \\ Houston, TX, USA; dDepartment of Infectious Diseases, University of Texas MD Anderson \\ Cancer Center, Houston, TX, USA
}

\section{Keywords}

Infarction · Escherichia coli · Meningitis · Vasculopathy

\begin{abstract}
Cerebral ischemia is a known complication of meningitis. Most Escherichia coli meningitisinduced infarcts have been reported in the neonatal and pediatric population. To the best of our knowledge, there have been no previous reports describing bilateral cortical infarcts in an adult secondary to a $\mathrm{K} 1$ strain of $E$. coli meningitis, and herein we report a case in a 25 -yearold female. The challenge in treating this patient was determining the duration of systemic antibiotic treatment and whether or not to use steroids. This study demonstrates the necessity of early diagnosis and treatment of $E$. coli meningitis to prevent neurological complications, including stroke.
\end{abstract}

(C) 2021 The Author(s).

Published by S. Karger AG, Basel

\section{Introduction}

Bacterial meningitis is one of the leading causes of infection-related death worldwide with high mortality rates up to $20 \%$ despite the availability of antibiotic therapy and acute neurological care [1]. Acute stroke occurs in 15-20\% of adult patients with bacterial meningitis often secondary to vasculitis [1]. Julayanont et al. [2] noted 3 phases in the pathogenesis of bacterial meningitis-induced cerebral angiopathy: (1) the infectious process in the subarachnoid space results in vasculitis and vasospasm, (2) followed by myonecrosis of the 
vessel wall which leads to vasodilation, and (3) then subendothelial edema and proliferation of smooth muscle develops which produces vascular stenosis and cerebral infarcts.

Studies have shown meningitis-induced infarcts have a predilection for the basal ganglia. Tai et al. [3] determined that tuberculous meningitis-induced infarcts were most commonly found in the basal ganglia (49\%), which are typically supplied by the lateral lenticulostriate arteries. However, infarcts in the cortical areas (18\%) are far less common. Vela-Duarte et al. [4] also reported a high percentage (75\%) of basal ganglia lacunar infarcts secondary to cryptococcal meningitis.

Among Gram-negative bacillary organisms, Escherichia coli is the most prevalent [5]. Of these E. coli strains, the K1 isolate accounts for approximately $80 \%$ of bacterial meningitis in neonates and infants; however, it is exceedingly rare in adults [5]. To the best of our knowledge, there are no previous reports of bilateral hemispheric cortical infarcts in an adult secondary to E. coli $\mathrm{K} 1$ meningitis.

\section{Case Presentation}

A 25-year-old Hispanic female presented to our emergency center with complaints of sudden onset severe neck pain, headache, and a fever of $101.4^{\circ} \mathrm{F}$ at home. She had a history of sacral myofibroblastic sarcoma and underwent a remote sacrectomy with lumbopelvic fixation as a child. She had a complicated course involving multiple hardware revisions at the L2-L4 levels due to wound breakdown. Two weeks prior to presentation, she underwent iliac screw revision and placement of an intrathecal pain pump.

In the emergency center, she was somnolent but without focal motor deficits. She developed a new-onset seizure for which she was given one dose each of lorazepam $2 \mathrm{mg} / \mathrm{mL}$ IV and levetiracetam 1,500 mg IV. A head CT revealed nonspecific hypodensities in the right frontal lobe, left parietal lobe, and left cerebellum. A lumbar puncture was performed, and broad-spectrum antibiotics were initiated for suspected bacterial meningitis. She was admitted to the intensive care unit and intubated due to a depressed level of consciousness. Electroencephalogram showed mild generalized slowing with left temporal dysfunction but no epileptiform discharges.

Cerebrospinal fluid (CSF) examination revealed a WBC count of $5,781 \mathrm{~mm}^{3}$, protein $578 \mathrm{mg} / \mathrm{dL}$, and glucose $<30 \mathrm{mg} / \mathrm{dL}$; Gram-negative rods were seen on stains, and a CSF multiplexed nucleic acid amplification test (NAAT) was positive for E. coli, and a BioFire CNS multiplex panel detected the K1 strain of E. coli. Corticosteroids were not administered. She underwent intrathecal pump removal, and the wound culture around the pump insertion revealed E. coli and secondarily Candida parapsilosis which was treated with fluconazole.

One day after admission, an MRI brain with and without contrast was obtained which demonstrated multifocal, bilateral cerebral and cerebellar cortically based nonenhancing diffusion restricting lesions consistent with cortical infarcts (Fig. 1, 2), which correlated with the hypodensities seen on the prior CT head. There was MR susceptibility noted within the cortical infarcts compatible with microhemorrhage. There was no leptomeningeal enhancement within the cisterns or sulci to support meningitis by imaging standards. A stroke workup was pursued including CT angiogram (CTA) of the head/neck which showed no arterial occlusion, stenosis, or dissection. There was no vasospasm of the circle of Willis vessels. A transthoracic echocardiogram was also unremarkable. A transesophageal echocardiogram was not pursued as the patient did not fulfill Duke's criteria for endocarditis. An MRI brain venogram revealed no dural venous sinus thrombosis. Following this extensive workup, the cortical infarcts were determined to be secondary to E. coli K1 meningitisinduced vasculopathy.

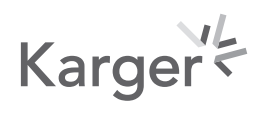



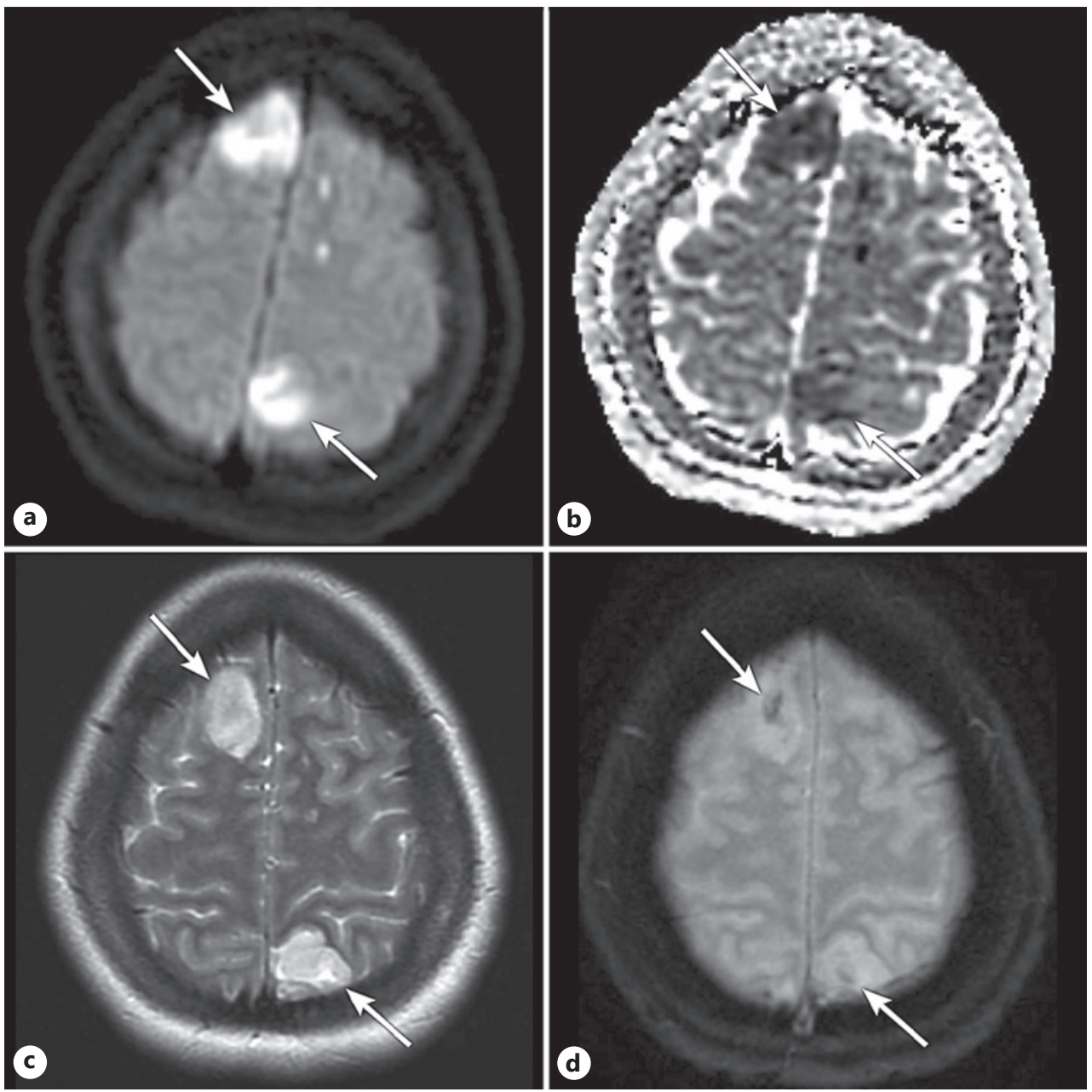

Fig. 1. MRI brain demonstrates large cortically based infarcts in the right superior frontal gyrus and left superior parietal lobule on DWI (a) and on ADC (b) in a $25 \mathrm{y} / \mathrm{o}$ female patient. The cortically based infarcts also show a gyriform pattern on T2 weighted imaging (c) and a small amount of susceptibility consistent with microhemorrhage on gradient echo imaging (d). MRI, magnetic resonance imaging; DWI, diffusion weighted imaging; ADC, apparent diffusion coefficient.

On day 4 of admission, the patient was successfully extubated. At this time, the patient was neurologically intact except for mild encephalopathy with no focal findings. Meropenem was changed to ceftriaxone at meningeal doses for 14 days with plans to continue suppressive therapy with $400 \mathrm{mg}$ cefpodoxine twice daily for hardware infection. The patient was discharged on day 16 after admission with phenytoin, ceftriaxone, and cefpodoxime. Four days later, phenytoin was stopped. Overall, the patient made a good recovery with no neurological deficits.

\section{Discussion/Conclusion}

We describe a previously unreported case of multiple bilateral cortical infarcts secondary to a $\mathrm{K} 1$ strain of $E$. coli meningitis in an adult with an infected intrathecal pump due to fecal contamination of the sacral wound. E. coli $\mathrm{K} 1$ in adults is rare and generally limited to exposure 

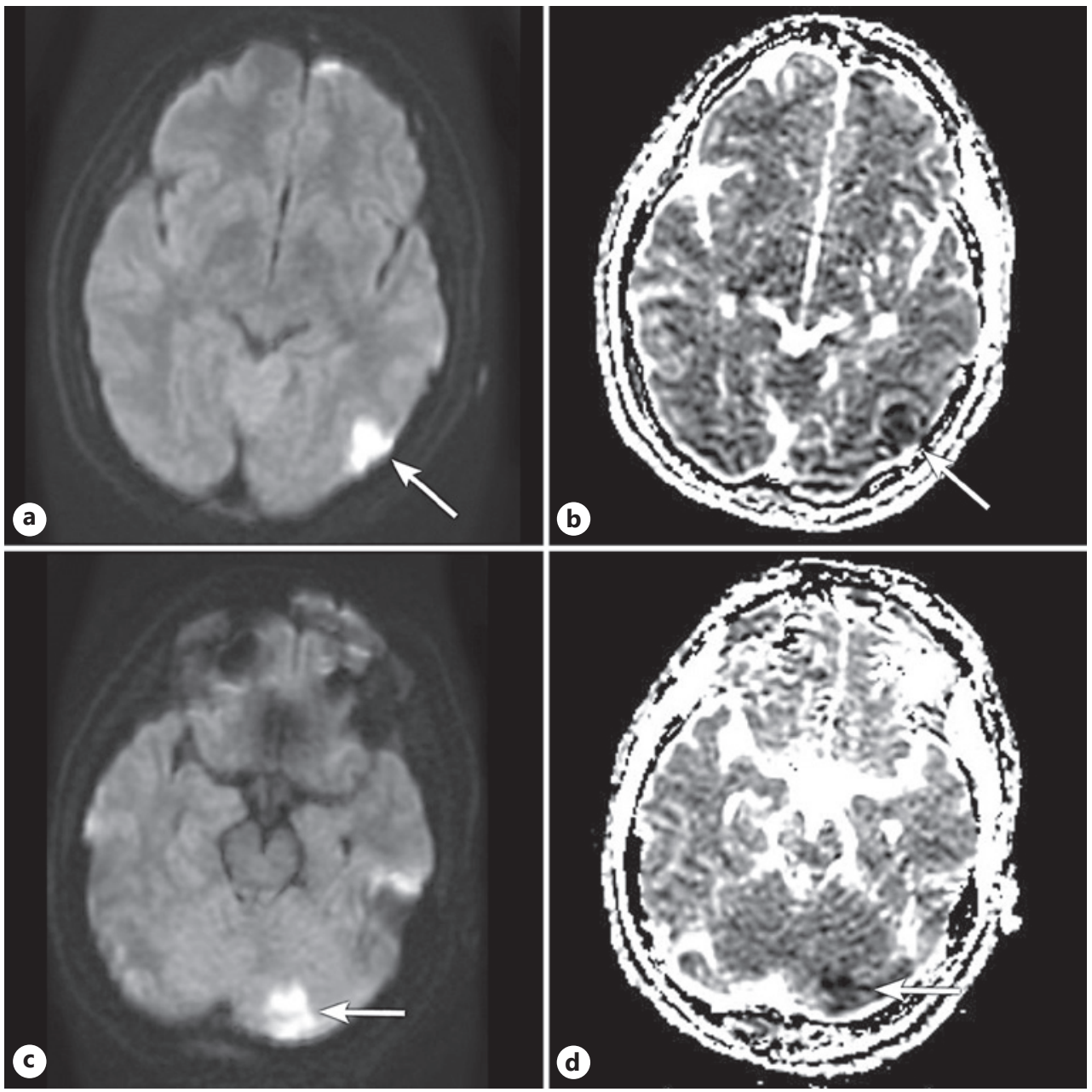

Fig. 2. MRI brain in the same patient also demonstrates cortical infarcts in the left occipital lobe on DWI (a) and ADC (b) and in the left cerebellum on DWI (c) and ADC (d). MRI, magnetic resonance imaging; DWI, diffusion weighted imaging; $\mathrm{ADC}$, apparent diffusion coefficient.

of sterile organs to the gastrointestinal tract after trauma or surgical interventions [6, 7]. The progression of $E$. coli $\mathrm{K} 1$ meningitis is often rapid, and it has a high mortality rate in adults (28-36\%) in the absence of proper antibiotic treatment [6, 7].

Early in our workup, we also considered septic emboli as a potential cause for the cerebral infarctions. However, there were no cardiac or carotid abnormalities to support septic emboli. The infarction patterns found in this case were also atypical for septic emboli which commonly appear as multiple bilateral infarcts in the cerebral gray-white matter junction [8]. This is an important distinction as vasculopathy-induced and septic emboli-induced infarct treatment regimens differ substantially. Standard treatment for embolic stroke includes anticoagulants, thrombolytic agents, and thrombectomy $[9,10]$. However, the role of anticoagulation therapies specifically treating infarcts secondary to bacterial meningitis is still unclear and requires further studies [2].

Diagnosis of our patient followed standard meningitis guidelines outlined by the Infections Diseases Society of America (IDSA) [11]. Standard treatment antimicrobial therapy for meningitis is third-generation cephalosporins with alternatives such as meropenem and ceftriaxone with recommended dosages of $6 \mathrm{~g}$ and $4 \mathrm{~g}$, respectively [11]. Our patient was given 
an antibiotic course of meropenem $2 \mathrm{~g}$ and fluconazole $800 \mathrm{mg}$ intravenously. Meropenem was later switched to ceftriaxone $2 \mathrm{~g}$. Because the patient responded well to the appropriate antibiotic therapy, adjuvant corticosteroid therapy was not given. Corticosteroid therapy has been shown to reduce the inflammatory response commonly seen in meningitis [12]. For example, it has been shown to decrease the complications associated with pneumococcal meningitis [13]. However, the utility of corticosteroid therapy in the setting of $E$. coli meningitis is not clear despite potential benefits in treating vasculopathy, so it was decided at the onset not to administer corticosteroids. Also, meningeal biopsy was not considered as the presumptive diagnosis of $E$. coli meningitis (K1 strain) was made based on the BioFire CNS multiplex panel result. If the patient's vasculopathy had not responded to antibiotics, the admitting Neurosurgeon had considered corticosteroid use and possible meningeal biopsy.

Adult E. coli $\mathrm{K} 1$ meningitis is a rare disease that requires rapid diagnosis and implementation of effective treatment. As with other causes of bacterial meningitis, a delay in diagnosis and therapy can result in death or chronic neurological sequelae. This report describes an unusual case of bilateral cortical infarcts in an adult patient with E. coli $\mathrm{K} 1$ meningitis-induced vasculopathy which was diagnosed early and successfully treated with an appropriate antibiotic course.

\section{Statement of Ethics}

This study involving a human participant was in accordance with the ethical standards of the Helsinki Declaration. Our institutional review board approved this retrospective single patient care report (IRB ID: 2020-1149) and granted a waiver of informed consent.

\section{Conflict of Interest Statement}

The authors report no conflicts of interest.

\section{Funding Sources}

The authors have no funding sources to declare. Specifically, funding was not needed to complete this case report.

\section{Author Contributions}

B.G., A.L.Y., and V.A.K. wrote the manuscript; B.J.B. and P.C.O. edited the manuscript. All authors read and approved the final manuscript.

\section{Data Availability Statement}

All the data generated and analyzed during this study have been included in this article. Further inquiries can be directed to the corresponding author. No data in this case report are openly available.

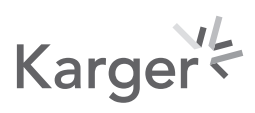


Case Reports in

Neurology

\begin{tabular}{l|l}
\hline Case Rep Neurol 2021;13:572-577 \\
\hline DOI: 10.1159/000518312 & $\begin{array}{l}\odot \text { 2021 The Author(s). Published by S. Karger AG, Basel } \\
\text { www.karger.com/crn }\end{array}$ \\
\hline
\end{tabular}

Gogia et al.: Cortical Infarcts in an Adult with E. coli K1 Meningitis

\section{References}

1 Murr C, Schonenberger S, Klein S, Bosel J. Complications of adult bacterial meningitis and their neurocritical care management: a case report and short review. J Neuroinfectious Dis. 2015;6(2):2-5.

2 Julayanont P, Ruthirago D, DeToledo JC. Bacterial meningitis and neurological complications in adults. Southwest Respir Crit Care Chron. 2016 Apr 4;4(14):5-16.

3 Tai MS, Viswanathan S, Rahmat K, Nor HM, Kadir KAA, Goh KJ, et al. Cerebral infarction pattern in tuberculous meningitis. Sci Rep. 2016;6(1):38802.

4 Vela-Duarte D, Nyberg E, Sillau S, Pate A, Castellanos P, Chastain DB, et al. Lacunar stroke in cryptococcal meningitis: clinical and radiographic features. J Stroke Cerebrovasc Dis. 2019 Jun;28(6):1767-72.

5 Kim KS. Human meningitis-associated Escherichia coli. EcoSal Plus. 2016 May;7(1).

6 Lu CH, Chang WN, Chuang YC, Chang HW. The prognostic factors of adult gram-negative bacillary meningitis. J Hosp Infect. 1998 Sep;40(1):27-34.

7 Gower DJ, Barrows AA 3rd, Kelly DL, Pegram S. Gram-negative bacillary meningitis in the adult: review of 39 cases. South Med J. 1986 Dec;79(12):1499-502.

8 Champey J, Pavese P, Bouvaist H, Kastler A, Krainik A, Francois P. Value of brain MRI in infective endocarditis: a narrative literature review. Eur J Clin Microbiol Infect Dis. 2015 Feb;35(2):159-68.

9 Kamel H, Healey JS. Cardioembolic stroke. Circ Res. 2017 Feb;120(3):514-26.

10 Ohya Y, Makihara N, Wakisaka K, Morita T, Ago T, Kitazono T, et al. Thrombolytic therapy in severe cardioembolic stroke after reversal of dabigatran with idarucizumab: case report and literature review. J Stroke Cerebrovasc Dis. 2018 Jul;27(7):e128-31.

11 Tunkel AR, Hartman BJ, Kaplan SL, Kaufman BA, Roos KL, Scheld WM, et al. Practice guidelines for the management of bacterial meningitis. Clin Infect Dis. 2004 Nov;39(9):1267-84.

12 Gundamraj S, Hasbun R. The use of adjunctive steroids in central nervous infections. Front Cell Infect Microbiol. 2020;10:592017.

13 van de Beek D, de Gans J, McIntyre P, Prasad K. Steroids in adults with acute bacterial meningitis: a systematic review. Lancet Infect Dis. 2004 Mar;4(3):139-43. 